\title{
A Flexible, (C)LP-based Approach to the Analysis of Object-Oriented Programs
}

\author{
Mario Méndez-Lojo ${ }^{1}$, Jorge Navas ${ }^{1}$, and Manuel V. Hermenegildo ${ }^{1,2}$ \\ 1 University of New Mexico (USA) \\ 2 Technical University of Madrid (Spain)
}

\begin{abstract}
Static analyses of object-oriented programs usually rely on intermediate representations that respect the original semantics while having a more uniform and basic syntax. Most of the work involving object-oriented languages and abstract interpretation usually omits the description of that language or just refers to the Control Flow Graph (CFG) it represents. However, this lack of formalization on one hand results in an absence of assurances regarding the correctness of the transformation and on the other it typically strongly couples the analysis to the source language. In this work we present a framework for analysis of object-oriented languages in which in a first phase we transform the input program into a representation based on Horn clauses. This allows on one hand proving the transformation correct attending to a simple condition and on the other being able to apply an existing analyzer for (constraint) logic programming to automatically derive a safe approximation of the semantics of the original program. The approach is flexible in the sense that the first phase decouples the analyzer from most languagedependent features, and correct because the set of Horn clauses returned by the transformation phase safely approximates the standard semantics of the input program. The resulting analysis is also reasonably scalable due to the use of mature, modular (C)LP-based analyzers. The overall approach allows us to report results for medium-sized programs.
\end{abstract}

\section{Introduction}

Analysis of object-oriented languages using abstract interpretation [10] is currently the subject of significant research (see, e.g., [21] and its references). The abstract interpretation approach brings an interesting and useful combination of characteristics: it is automatic and practical, producing useful results for a good number of applications, while at the same time being rigorous and semanticsbased. The gap between programs and semantics is greater in the case of objectoriented languages than in, for example, declarative languages. For this reason, static analyses of object-oriented programs usually rely on intermediate languages that respect the original semantics while having a more uniform and basic syntax (e.g., block-based representations) and a more declarative semantics (e.g., static single assignment transformations). Some significant concrete examples which have been proposed of such intermediate representations for object-oriented programs are Jimple [31] for Java or BoogiePL [11] for .NET. 
In this paper we propose the use of a Horn clause-based representation as an intermediate language. Our objective is twofold. On one hand we would like to take advantage of existing analyzers for (constraint) logic programs. On the other, we want to be able to offer assurances that the output of the process of transformation into the intermediate representation safely approximates the standard semantics of the input program. Performing the analysis using logic programming tools offers a number of advantages, such as the relative maturity and sophistication of the solutions available, like abstract interpreters $[13,16]$ (which offer parametric, efficient, and modular fixpoint algorithms) and verifiers. A second strength of our transformational approach is that the framework can be easily adapted to the analysis of other languages without having to redefine the fixpoint algorithm [23]. In fact, using the intermediate representation that we propose, from the analyzer point of view an object-oriented program is indistinguishable from, e.g., a Prolog one (although of course different abstract domains and definitions of pseudo-builtins are used). This brings in the additional advantage of being able to analyze multiple languages within the same framework.

We start by describing our methodology (Section 2) and our approach to ensuring correctness using some fundamental parts of the transformation of Java programs into our representation as examples (Section 3). Section 4 shows how analysis of specific aspects of Java can be optimized using metainformation. We then illustrate the application of our approach to other languages, such as C\# (Section 5). We also report on an implementation of the ideas presented in this paper using the abstract interpretation-based CiaoPP [16] framework. It can be configured for many different analyses by simply plugging the corresponding abstract domain. The examples try to detect null pointer dereferences (nullity analysis) and eliminate dynamic dispatch (class analysis) in Java programs. The experiments in Section 6 show that the technique scales well in non trivial scenarios, and results in smaller analysis times than similar previous work. Related abstract interpretation-based frameworks, and how they differ from ours, are discussed in Section 7 , and Section 8 presents our conclusions.

\section{Methodology}

Our framework is composed of a front-end preprocessor and a back-end analyzer, as shown in Figure 1. The preprocessor transforms an input in Java source format into a set of Horn clauses that represent a safe approximation of its standard semantics (Sect 3). Sometimes the source code is not available, so we also accept Java bytecode as a valid input format. In this case the (de)compilation from bytecode to Horn clauses is based on a postprocessing of the Jimple representation returned by the Soot [31] tool. It is beyond the scope of this paper to provide a detailed description of this particular transformation; the reader is referred to [23] (which presents the transformation and a specific fixpoint algorithm) for details. In both cases the same subset of the language is covered by the framework. Our ultimate objective is to support the full Java language 


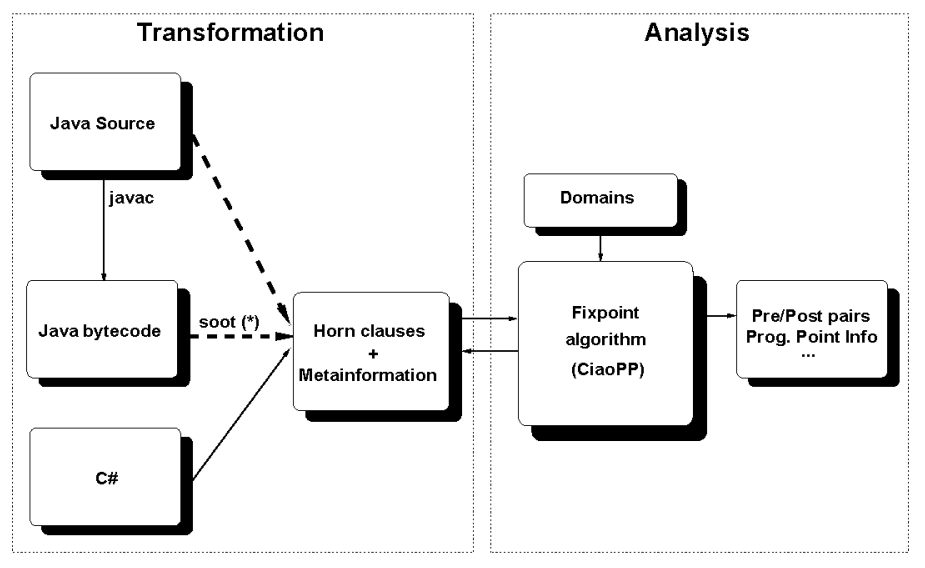

Fig. 1. Pipeline of transformation and analysis

but the current implementation has some limitations: it does not support dynamic loading of classes, threads, or runtime exceptions. Also, analysis of the JDK libraries is done under a worst-case assumption. We have distinguished in the figure the two implemented transformations with dotted arrows to clearly separate them from already existing phases.

Different languages can be incorporated into the framework (i.e., analyzed) by providing a correct transformation for them. For example, programs written in Ciao are obviously also accepted by the system as input. Compatibility with other object-oriented languages written in languages like $\mathrm{C \#}$, that share many syntactic and semantics features with Java, is easily achievable as illustrated in Section 5 .

The resulting Horn-clause intermediate representation is then analyzed using the CiaoPP framework [16] and benefits from its advanced features: efficient computation of fixpoints using memoization, context-sensitivity, modularity, etc. The programmer needs only to implement (in Ciao) the particular abstract domain of interest, which includes also defining the abstract meaning of a set of "built-in" predicates that represent the language-dependent semantics of the basic operations of the source language. On the other hand, our approach does liberate the designer of an analysis from the burden of coding a fast, reliable, and efficient abstract interpretation platform. Analysis results are given in the standard form $(p, \sigma)$, where $p$ uniquely identifies a program point and $\sigma$ is an abstract state which safely approximates all the possible states at that program point during runtime. Metainformation computed during the transformation process allows relating those line numbers with the ones of the original bytecode or source program, making it possible to reflect back the results on the original program text (as JML-like assertions [18]), pinpoint errors in the original program, or implement compiler optimizations. 


\section{Correctness of the transformation phase}

Our Horn clause representation of a Java program is basically an unfolded, three-address version of the source where the operational semantics of some instructions is made explicit. The transformed code is denoted by the $c$ subindex: for example, the result of transforming a virtual invocation $v \cdot \mathrm{m}\left(v_{1}, \ldots, v_{n}\right)$ is $v_{c} \cdot \mathrm{m}_{c}\left(v_{1_{c}}, \ldots, v_{n_{c}}\right)=v \cdot \mathrm{m}_{c}\left(v_{1}, \ldots, v_{n}\right)$, since variable expressions are not transformed $\left(v_{c}=v\right)$.

Correctness of the transformation requires that the original program prog be emulated by $\operatorname{prog}_{\mathcal{C}}$ thus $\mathcal{C} \|$ prog $\rrbracket=\mathcal{C} \llbracket$ prog $g_{c}$, where the semantics operator $\mathcal{C} \llbracket \|$ com $\mapsto(\mathcal{D} \mapsto \mathcal{D})$ takes as input a command com and a concrete state, and returns the output state. The operator has been defined in [15] and (from a denotational point of view) in $[2,29]$. Correctness of the preprocessing and analysis requires that if the set of Horn clauses program is safely approximated (using a given abstract domain) by the analysis, so is the original: $\mathcal{C}^{*} \llbracket$ prog $\rrbracket=$ $\mathcal{C}^{*} \llbracket$ prog $_{c} \rrbracket$. The operator $\mathcal{C}^{*} \llbracket \rrbracket: \operatorname{com} \mapsto\left(\mathcal{D}^{*} \mapsto \mathcal{D}^{*}\right)$ is the abstract counterpart of $\mathcal{C} \llbracket$.

We will take a slightly different approach by interpreting Java semantics as a particular case of SLD [17] resolution, in which the computation rule in use is left-to-right (commands are executed in the order they appear in the program) and the search rule used to determine the target method in an invocation does not really matter, since execution of the Java program is deterministic and therefore for any literal there is exactly one clause that unifies with it at runtime. Therefore, if $\mathcal{S} \llbracket: c o m \mapsto(\mathcal{D} \mapsto \mathcal{P}(\mathcal{D}))$ is the SLD semantics operator, the condition $\mathcal{S} \llbracket$ prog $\rrbracket=\{\mathcal{C} \llbracket$ prog $\rrbracket\}$ ensures $\mathcal{S}^{*} \llbracket$ prog $\rrbracket=\mathcal{C}^{*} \llbracket$ prog $\rrbracket$. Again, $\mathcal{S}^{*} \llbracket \rrbracket: c o m \mapsto\left(\mathcal{D}^{*} \mapsto \mathcal{D}^{*}\right)$ is the (collecting) abstract version of $\mathcal{S} \llbracket \rrbracket$.

This formalization is useful since it helps in understanding the Java source as a set of Horn clauses (methods) composed by zero or more goals, the commands. It is also helpful because our transformation introduces new clauses such that now more than one clause might unify with a given literal. This is equivalent to saying that the execution of the transformed program on some input state might result in multiple output states, of which one is the unique state that the original program would return: $\mathcal{S} \llbracket p r o g \rrbracket \subseteq \mathcal{S} \llbracket$ prog $_{c} \rrbracket$. An interesting property of that transformed program is that its abstract semantics $\mathcal{S}^{*} \llbracket p r o g_{c} \rrbracket$ still correctly approximates that of the original, i.e., $\mathcal{S}^{*} \llbracket p r o g \rrbracket \leq \mathcal{S}^{*} \llbracket p r o g_{c} \rrbracket$. Therefore, all we have to prove in order to show that the results of the analysis are correct is that $\mathcal{S} \llbracket p r o g \rrbracket \subseteq \mathcal{S} \llbracket p r o g_{c} \rrbracket$ (or $\mathcal{C} \llbracket$ prog $\rrbracket \in \mathcal{S} \llbracket \operatorname{prog}_{c} \rrbracket$ ) holds. Space limitations prevent us from including the proofs for the whole transformation algorithm. Instead, we provide the proof for the case of the virtual invocations expression, which is one of the most complex operations supported.

\subsection{Correctness of a virtual invocation}

The description of the standard semantics in this section is a slightly simplified version of the more formal specification described in [29]. We distinguish between two different kinds of invocations: virtual and static. Assume that calls 

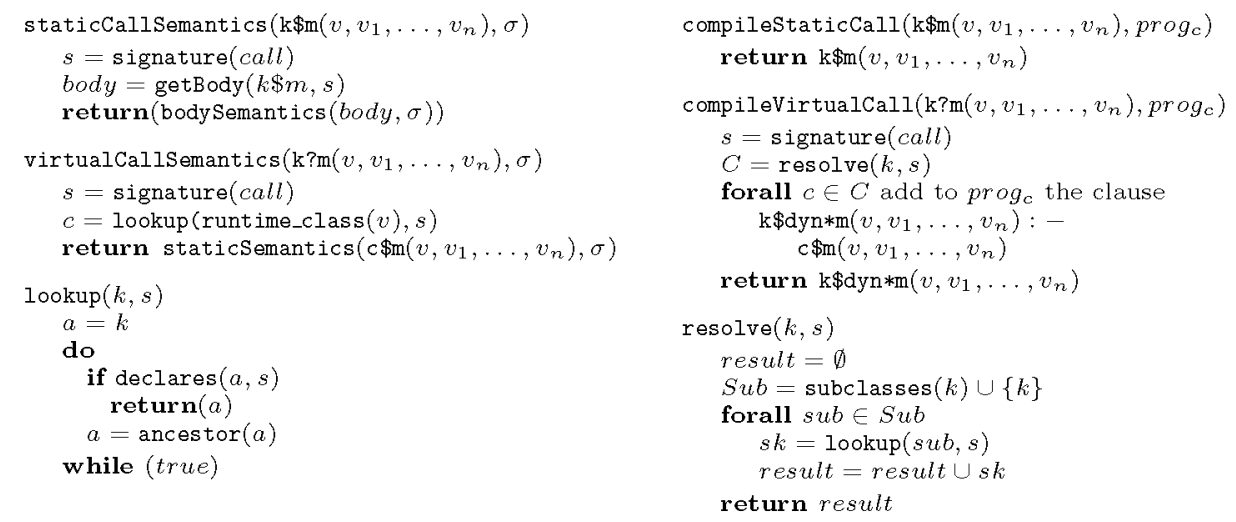

Fig. 2. Standard semantics (left) and transformation (right) of method calls

of the first type have been rewritten as $\mathrm{k} ? \mathrm{~m}\left(v, v_{1}, \ldots, v_{n}\right)$ and the static ones as $\operatorname{k} \$ \mathrm{~m}\left(v, v_{1}, \ldots, v_{n}\right)$, where $k$ is the declared type of $v$. Note that we rewrote the call syntax so the invoked object $v$ is now the first actual parameter. The main difference between the two is that while in virtual invocations we need to figure out the particular class of $v$ through a lookup in the class hierarchy, that operation is unnecessary in static calls since there is only one possible receiver.

In the left column of Figure 2 we present the pseudocode for the semantics of a static call (here denoted by staticCallSemantics) and a virtual call (here denoted by virtualCallSemantics). The particular signature of the invocation has to be calculated in order to distinguish which implementation to choose, since in Java (as in the Horn clauses) there can be many methods with the same name and arity, but here they will differ in the type of at least one of the formal parameters. Also, we will assume that there exists a function runtime_class that returns the runtime type of the object passed as parameter.

We refer to the tuple $\left(v, v_{1}, \ldots, v_{n}\right)$ as pars. The standard semantics of the call in the original program is $\mathcal{C} \llbracket k ? \mathrm{~m}($ pars $) \rrbracket \sigma=\mathcal{C} \llbracket c \$ \mathrm{~m}($ pars $) \rrbracket \sigma$, where $c$ is the value returned by lookup(runtime_class $(v), s)$. The SLD semantics of the transformed version is $\mathcal{S} \llbracket k ? \mathrm{~m}_{c}($ pars $) \rrbracket \sigma$, which the transformation ensures to be $\mathcal{S} \llbracket k \$ \operatorname{dyn} * \mathrm{~m}($ pars $) \rrbracket \sigma=\bigcup_{i} \mathcal{S} \llbracket c_{i} \$ \mathrm{~m}($ par $s) \rrbracket \sigma$, where $c_{i} \in \operatorname{resolve}(k, s)$. The correctness condition is now reduced to proving that $c$ is equal to some $c_{i}$. This is equivalent to showing that lookup(runtime_class $(v), s) \in \operatorname{resolve}(k, s)$, which can be further rewritten as lookup(runtime_class $(v), s) \in\{$ lookup $(s u b, s) \mid s u b \in$ subclasses $(k) \cup\{k\}\}$. But the runtime type of $v$ can only be $k$ or a subclass of it in a type safe language as Java, and therefore the condition always holds.

Example 1. Assume a hierarchy of classes like in Figure 3. The root class A declares a method foo which is further redefined (overwritten) in subclasses $B$, $\mathrm{C}$, and $\mathrm{Z}$. If the original program in Figure $3 \mathrm{a}$ ) contains a virtual invocation to foo in an instance declared as being of class X, our compiler automatically 

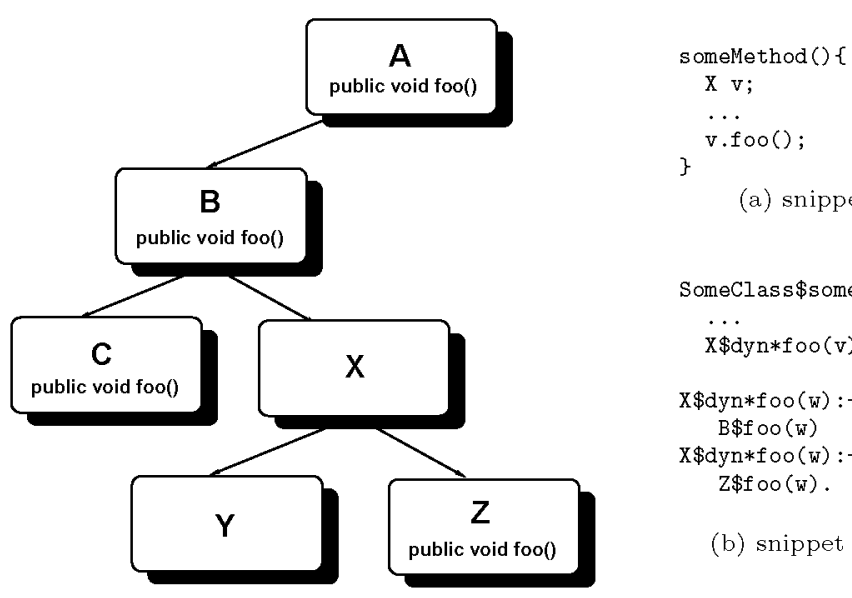

(a) snippet of the original program

SomeClass \$someMethod() :-

...

$\mathrm{X} \$ \mathrm{dyn} * \mathrm{foo}(\mathrm{v})$.

$\mathrm{X} \$ \mathrm{dyn} * f \circ o(\mathrm{~W}):-$

$B \$ f \circ o(w)$

$\mathrm{X} \$ \mathrm{dyn} * \mathrm{foo}(\mathrm{w}):-$

$\mathrm{Z} \$ \mathrm{f} 0 \mathrm{o}(\mathrm{w})$.

(b) snippet of the transformed program

Fig. 3. Transformation of a virtual invocation

transforms it into a call to a new method with two new clauses (methods) that represent all the possible receiver implementations for the call. Because $\mathrm{X}$ is a direct subclass of $B$, it can never inherit the original $A$ implementation but only the $B$ one, represented by the first clause of $x \$ d y n \$ f o o$. Alternatively, any object of type $\mathrm{Y}$ and $\mathrm{Z}$ is also of type $\mathrm{X}$ and therefore we include a call to the $\mathrm{Z}$ version of foo in the second clause. The $C$ implementation is discarded because of type incompatibility.

The process described has many interesting properties. First, it is based on assuming SLD resolution semantics for the transformed Horn clause program. This allows reusing existing analyzers without having to redefine the abstract unification operator in order to deal with language-dependent features, as in the case of virtual invocation. We implemented our Java analyses on top of the CiaoPP Prolog analyzer [16] without modifying its code, even when specific abstract domains and "builtin" definitions for Java language constructs had to be provided. A second strength is that correctness of the transformation depends only on showing that $\mathcal{C} \llbracket$ comm $\rrbracket \in \mathcal{S} \llbracket \mathrm{comm}_{c} \rrbracket$ holds for every command (and expression) in the source language. Although not trivial, the proof can be slightly modified for similar languages to Java, so neither the compiler nor the abstract domains need to be completely rewritten. In the case of Ciao, the proof is trivial since prog $_{c}=$ prog.

\section{Metainformation}

Full independence from the language cannot be achieved only through program transformations. Sometimes the fixpoint algorithm can be optimized if some characteristics related to the original source are known. In other occasions the abstract domain needs information about the program that cannot be found in 


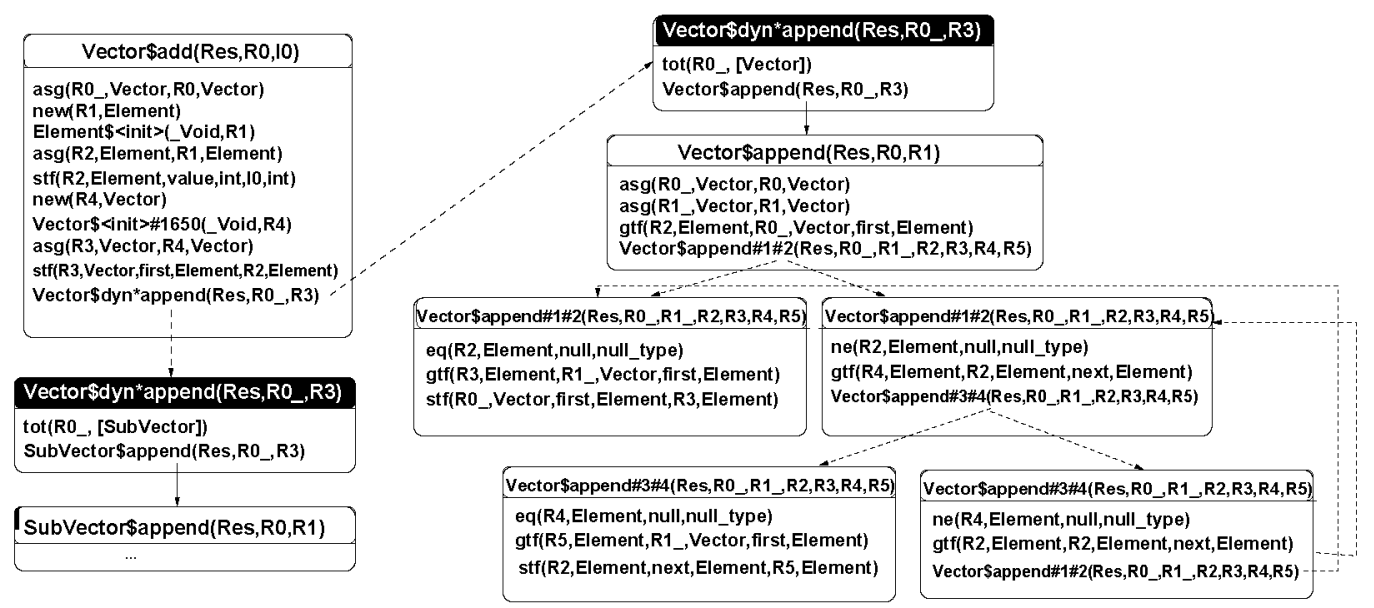

Fig. 5. Call Graph for the example in Figure 4

are indistinguishable from the caller's point of view, thus causing invocations to the method to be non-deterministic (i.e., causing the execution of one clause or another). Entry clauses are marked in grey, internal ones in white; dotted arrows denote non-deterministic flows while the continuous ones symbolize deterministic calls.

Another flow transformation (extra clauses) tries to expose the internal structure of some complex Java features, which sometimes encode sophisticated operations. That is the case of the virtual invocations studied in Section 3. Coming back to the example in Figure 4, note that the call to append within add is polymorphic: it might execute the implementation in Vector or the one in SubVector. We make this semantics explicit by inspecting the application hierarchy and replacing the virtual invocation with a set of resolved calls, one for each possible implementation. The method acting as a "hub" is called an extra clause; in the example we have two, Vector\$dyn*append, marked in black. They behave in a very similar way to the conditional discussed previously, since the program flow might go through two alternative paths (clauses), one for each implementation of append. Each branch contains a guard (tot, see the first statement in each of the Vector $\$ d y n *$ append clauses) listing the acceptable types for the callee.

It is interesting how, in an analogous way to the clause case, we introduced extra statements to further simplify analysis. For example, the mentioned tot (type of this) builtin filters the execution of subsequent statements when the class of the instance is not listed in the set of possibilities; guard statements have a similar goal in clauses that come from conditional constructions. In Figure 5 the eq call at the beginning of the leftmost Vector\$append\#1\#2 clause refers to the condition for executing the first branch, while the ne call contains its negated version, for the second alternative. Also, those methods that are entry but not extra contain assignments to shadow variables that simulate the call-by-reference semantics [23]. 


\begin{tabular}{|l|r|r|r|r|r|}
\hline name & $k$ & $m$ & $i$ & $p p$ & $c t$ \\
\hline jolden.health.Health & 8 & 30 & 637 & 933 & 1.1 \\
jolden.bh.BH & 9 & 70 & 1208 & 1739 & 3.2 \\
jolden.voronoi. Voronoi & 6 & 73 & 988 & 1340 & 2.2 \\
jolden.mst.MST & 6 & 36 & 445 & 665 & 0.1 \\
jolden.power.Power & 6 & 32 & 1017 & 1270 & 2.1 \\
jolden.treeadd. TreeAdd & 2 & 12 & 193 & 274 & 2.0 \\
jolden.em3d.Em3d & 4 & 22 & 447 & 669 & 0.1 \\
jolden.perimeter.Perimeter & 10 & 45 & 543 & 814 & 0.1 \\
jolden.bisort.BiSort & 2 & 15 & 323 & 476 & 0.1 \\
jolden.all.All & 50 & 317 & 5839 & 7251 & 11.0 \\
\hline
\end{tabular}

Fig. 8. Statistics of the transformation phase.

are present in the Horn clause program analyzed. This metric slightly differs from the number of instructions in the sense that extra clauses and builtins make it somewhat larger; $p p$ also provides a better approximation of the size and complexity of the program analyzed because the semantics of the objectoriented program is made explicit, as seen in Section 2. The fifth column (ct) shows the time invested (given in seconds) in transforming the input program and producing the Horn clause version and the metainformation.

The second experiment shown in Figure 9 illustrates the scalability, efficiency, and precision of the analysis component of our framework. We first use a simple abstract domain, Nullity, capable of approximating which variables are definitely null and which ones definitely point to a non-null location. The second abstract domain is a Class Hierarchy Analysis [3], which uses the combination of the statically declared type of an object and the class hierarchy of the program to determine the set of possible targets of a virtual invocation. The use of a Class Hierarchy Analysis shows the scalability of our framework for a domain with non-linear worst-case complexity in its operations. Additionally, it also reflects the usefulness of metainformation files since they are required by the CHA domain in order to access the hiercharchy tree. The columns labeled $p p^{\prime}$ show the number of program points reachable by the analyses. Therefore, $p p^{\prime}$ may differ from $p p$ because the number of analyzed program points is not always the total number of program points in the program: some commands are found to be unreachable. Since our framework is multivariant and can thus keep track of different contexts at each program point, at the end of analysis there may be more than one abstract state associated with each program point. Thus, the number of abstract states is typically larger than the number of reachable program points. Columns ast provide the total number of these abstract states inferred by analyses. The level of multivariance is the ratio $a s t / p p^{\prime}$, presented in columns st. In general, such a larger number for st tends to indicate more precise results. Running times are listed in columns pt (time invested in preprocessing the program which includes the extraction of metainformation for each method in the Horn clause program and the construction of the class hierarchy) and at (analysis time); both are also given in seconds.

The benchmarks have been tested in both experiments on a Pentium $M$ 1.73Ghz with $1 \mathrm{~Gb}$ of RAM, and averaging several runs after eliminating the best and worst values. We chose to show separately the total times of the two phases 


\begin{tabular}{|c|c|c|c|c|c|c|c|c|c|}
\hline & & \multicolumn{4}{|c|}{ Nullity } & \multicolumn{4}{|c|}{ CHA } \\
\hline & $p t$ & $p p^{\prime}$ & $a s t$ & $s t$ & $a t$ & $p p^{\prime}$ & $a s t$ & $s t$ & $a t$ \\
\hline jolden.health. Health & 2.1 & 921 & 5836 & 6.3 & 9.6 & 933 & 3542 & 3.8 & 52.1 \\
\hline jolden.bh.BH & 2.2 & 1739 & 12384 & 7.1 & 50.1 & 1739 & 4757 & 2.7 & 59.4 \\
\hline jolden.voronoi.Voronoi & 2.2 & 1277 & 5492 & 4.3 & 11.5 & 1340 & 5147 & 3.8 & 81.3 \\
\hline jolden.mst.MST & 2.1 & 496 & 1503 & 3.0 & 1.1 & 665 & 1609 & 2.4 & 11.6 \\
\hline jolden.power.Power & 2.1 & 1270 & 10560 & 8.3 & 29.9 & 1270 & 2908 & 2.3 & 32.7 \\
\hline jolden.treeadd.TreeAdd & 2.0 & 274 & 880 & 3.2 & 0.6 & 274 & 729 & 2.6 & 6.1 \\
\hline jolden.em3d. Em3d & 2.0 & 669 & 5565 & 8.3 & 0.9 & 669 & 3320 & 4.9 & 49.5 \\
\hline jolden.perimeter. Perimeter & 2.1 & 814 & 2653 & 3.2 & 1.7 & 814 & 3731 & 4.5 & 25.0 \\
\hline jolden.bisort.BiSort & 2.1 & 476 & 3353 & 7.0 & 5.8 & 476 & 1614 & 3.4 & 15.6 \\
\hline jolden.all.All & 2.6 & 7188 & 48476 & 6.7 & 145.9 & 7251 & 29586 & 4.1 & 391.2 \\
\hline
\end{tabular}

Fig. 9. Statistics for the Nullity and Class Hierarchy (CHA) domains.

(transformation and analysis) because we expect the transformation process to be fully run only once. Later executions can use incremental compilation for those files that changed, so that the overhead of the preprocessing phase should be almost negligible in medium and large programs. Although the same approach can be taken for the analysis [27], the current implementation is not incremental.

\section{Related work}

Most of the previous research in analysis of object-oriented programs concentrates on finding new abstract domains that better approximate a particular concrete property of the program analyzed in order to optimize compilation (e.g., [4,28]) or statically verify certain properties about the runtime behavior of the code (e.g., $[14,19]$ ). In contrast to this concentration and progress on the development of new, refined domains there has been comparatively little work on the formal specification of the intermediate language to which the analyzed program is transformed or in the application of existing logic programming techniques. In [24] the authors describe how to automatically derive Prolog versions of Java programs that share the same operational semantics. However, the compilation applies to a smaller subset of Java than that supported in our work and no experimental results are provided. Also, the technique is presented from a more informal perspective and no analysis is attempted over the transformed logic programs.

More closely related to ours is the work presented in [1], which draws in part on the ideas of [25]. The authors also focus on how to reuse existing logic programming tools, in order to analyze Java bytecode. The approach is based on encoding an interpreter of the Java Virtual Machine bytecode in a logic language, Ciao [6], and then partially evaluating this interpreter with respect to the concrete program to be analyzed. This results in a residual program which has the same semantics as the original one but is often easier to analyze than the original set of bytecode+interpreter. As in our case, the analysis and verification experiments are performed using the CiaoPP [16] tool.

While the approach of [1] is obviously very interesting, it also has the shortcoming that it is quite dependent on the quality of the results obtained by the partial evaluator. Given the state of the art in partial evaluation, this clearly 
varies significantly depending on the input program. Our approach is based instead on a direct translation from the Java program into a Horn clause representation, which obviates this problem (at the cost of having to write and prove correct the transformer). Also, in our translation we do not try to mimic the operational semantics of the Java program in the Horn clause version (i.e., the resulting program if run, e.g., on a Prolog system, would not necessarily produce equivalent results to those of the Java program). Instead, our aim is to safely approximate the semantics of the Java program in the Horn clause representation by taking advantage of the (collecting) SLD semantics assumed by the analyzer. This allows flexibility in the translation and eliminates the burden of having to simulate exactly the operational semantics of the source language since we do not want to execute the program but only to obtain safe results by analyzing it. The flexibility and directness of our approach also allows us to support a much larger subset of the language than in [1], including many features such as exceptions, inheritance, interfaces, etc. Furthermore, since the fixpoint algorithm is sensitive to the size and characteristics of the intermediate representation, the fact that our direct translation guarantees a compact intermediate representation can arguably result in a more scalable solution. For example, in this work (see Section 6) we have been able to report on examples that are about twenty times larger and can be analyzed in less time.

In most of the (non CLP-based) abstract interpretation framework for analysis of Java (e.g., $[4,7])$ the authors prefer to focus on particular properties and therefore their solutions (abstract domains and analysis algorithms) are tied to them, even when if they may be explicitly labeled as multipurpose [20]. In [26] the authors use a framework that is closely related to Gaia [8]. However, the intermediate representation is not described and the semantics of the interprocedural operations is again tied to the Java language. Also, the benchmarks used are smaller than those that we report on. The more recent Julia framework [30] is intended to be generic from the point of view of domains but once more also targets Java as unique source language. This framework is capable of analyzing large programs in a top-down way, as in our approach, the main other difference being that we support multivariance, inherited from the CiaoPP analyzer. Finally, in [22] another interesting generic static analyzer for the modular analysis and verification of Java classes is presented. The algorithm presented is also top down but is again tailored specifically to Java source.

\section{Conclusions and future work}

We have presented a transformation-based framework for analysis of objectoriented programs, which is generic in terms of the source language and abstract domain in use. The framework consists of a two-step process: a transformation of the program into a set of Horn clauses that represents a correct approximation of its standard semantics, and a mature and sophisticated fixpoint algorithm. We claim that our approach is flexible in the sense that the first phase decouples the fixpoint algorithm from any language-dependent feature. Furthermore, 
our experimental evaluations support the scalability of our framework showing results for medium-sized programs as well as its efficiency analyzing them in a reasonable amount of time, and precision showing high rates of multivariance.

We have performed some promising experiments on an ample subset of Java, as shown in this paper, but our aim is to support the full Java language. Also, we are currently incorporating more sophisticated abstract domains (e.g., pointsto/sharing analysis). Moreover, we expect to increase the scalability of our approach, analyzing larger programs than shown in this paper. To this end, we are studying the inclusion of modular and incremental features in our fixpoint algorithm.

\section{References}

1. E. Albert, M. Gómez-Zamalloa, L. Hubert, and G. Puebla. Verification of Java Bytecode using Analysis and Transformation of Logic Programs. In Ninth International Symposium on Practical Aspects of Declarative Languages, number 4354 in LNCS, pages 124-139. Springer-Verlag, January 2007.

2. Jim Alves-Foss, editor. Formal Syntax and Semantics of Java, volume 1523 of Lecture Notes in Computer Science. Springer, 1999.

3. David F. Bacon and Peter F. Sweeney. Fast static analysis of $\mathrm{c}++$ virtual function calls. In OOPSLA, pages 324-341, 1996.

4. Bruno Blanchet. Escape Analysis for Object Oriented Languages. Application to Java(TM). In Conference on Object-Oriented Programming, Systems, Languages and Applications (OOPSLA'99), pages 20-34, Denver, Colorado, November 1999.

5. M. Bruynooghe. A Practical Framework for the Abstract Interpretation of Logic Programs. Journal of Logic Programming, 10:91-124, 1991.

6. F. Bueno, D. Cabeza, M. Carro, M. Hermenegildo, P. López-García, and G. Puebla (Eds.). The Ciao System. Reference Manual (v1.10). Technical report, School of Computer Science (UPM), 2004. Available at http://ww. ciaohome.org.

7. Bor-Yuh Evan Chang and K. Rustan M. Leino. Abstract interpretation with alien expressions and heap structures. In VMCAI, pages 147-163, 2005.

8. B. Le Charlier and P. Van Hentenryck. Experimental Evaluation of a Generic Abstract Interpretation Algorithm for Prolog. ACM Transactions on Programming Languages and Systems, 16(1):35-101, 1994.

9. JOlden Suite Collection. http://ww-ali.cs.umass.edu/DaCapo/benchmarks . html.

10. P. Cousot and R. Cousot. Abstract Interpretation: a Unified Lattice Model for Static Analysis of Programs by Construction or Approximation of Fixpoints. In Fourth ACM Symposium on Principles of Programming Languages, pages 238-252, 1977.

11. Rob DeLine and K. Rustan M. Leino. BoogiePL: A typed procedural language for checking object-oriented programs. Technical Report MSR-TR-2005-70, Microsoft Research, 2005.

12. S. W. Dietrich. Extension Tables: Memo Relations in Logic Programming. In Fourth IEEE Symposium on Logic Programming, pages 264-272, September 1987.

13. Christian Fecht. Gena - a tool for generating prolog analyzers from specifications. In SAS '95: Proceedings of the Second International Symposium on Static Analysis, pages 418-419, London, UK, 1995. Springer-Verlag. 
14. S. Genaim and F. Spoto. Information Flow Analysis for Java Bytecode. In Proc. of VMCAI, LNCS. Springer-Verlag, 2005.

15. James Gosling, Bill Joy, Guy Steele, and Gilad Bracha. Java(TM) Language Specification, The (3rd Edition) (Java (Addison-Wesley)). Addison-Wesley Professional, 2005.

16. M. Hermenegildo, G. Puebla, F. Bueno, and P. López-García. Program Development Using Abstract Interpretation (and The Ciao System Preprocessor). In Proc. of SAS'03, pages 127-152. Springer LNCS 2694, 2003.

17. R. Kowalski and D. Kuehner. Linear resolution with selection function. Artificial Intelligence, 2:227-260, 1971.

18. Gary T. Leavens, Albert L. Baker, and Clyde Ruby. Preliminary design of jml: a behavioral interface specification language for java. SIGSOFT Softw. Eng. Notes, $31(3): 1-38,2006$.

19. Xavier Leroy. Java bytecode verification: An overview. In $C A V$, pages 265-285, 2001.

20. Tal Lev-Ami and Shmuel Sagiv. TVLA: A system for implementing static analyses. In $S A S, 2000$.

21. F. Logozzo and A. Cortesi. Abstract interpretation and object-oriented languages: quo vadis? In Proc. of the 1st. Int'l. Workshop on Abstract Interpretation of Objectoriented Languages (AIOOL'05), ENTCS. Elsevier Science, January 2005.

22. Francesco Logozzo. Cibai: An abstract interpreation-based static analyzer for modular analysis and verification of java classes. In VMCAI'07. To appear, Jan 2007.

23. J. Navas, M. Méndez-Lojo, and M. Hermenegildo. An Efficient, Context and Path Sensitive Analysis Framework for Java Programs. In 9th Workshop on Formal Techniques for Java-like Programs FTfJP 2007, July 2007. To appear.

24. J. Peralta and J.Cruz-Carlon. From static single-assignment form to definite programs and back. Extended abstract in International Symposium on Logic-based Program Synthesis and Transformation (LOPSTR), July 2006.

25. J.C. Peralta, J. Gallagher, and H. Sağlam. Analysis of imperative programs through analysis of constraint logic programs. In G. Levi, editor, Static Analysis. 5th International Symposium, SAS'98, Pisa, volume 1503 of LNCS, pages 246-261, 1998.

26. Isabelle Pollet. Towards a generic framework for the abstract interpretation of Java. PhD thesis, Catholic University of Louvain, 2004. Dept. of Computer Science.

27. G. Puebla and M. Hermenegildo. Optimized Algorithms for the Incremental Analysis of Logic Programs. In International Static Analysis Symposium, number 1145 in LNCS, pages 270-284. Springer-Verlag, September 1996.

28. Erik Ruf. Effective synchronization removal for java. In $P L D I$, pages 208-218, 2000 .

29. Stefano Secci and Fausto Spoto. Pair-sharing analysis of object-oriented programs. In $S A S$, pages $320-335,2005$.

30. F. Spoto. Julia: A Generic Static Analyser for the Java Bytecode. In Proc. of the 7th Workshop on Formal Techniques for Java-like Programs, FTfJP'2005, Glasgow, Scotland, July 2005.

31. Raja Vallee-Rai, Laurie Hendren, Vijay Sundaresan, Patrick Lam, Etienne Gagnon, and Phong Co. Soot - a Java optimization framework. In Proceedings of CASCON 1999, pages 125-135, 1999. 